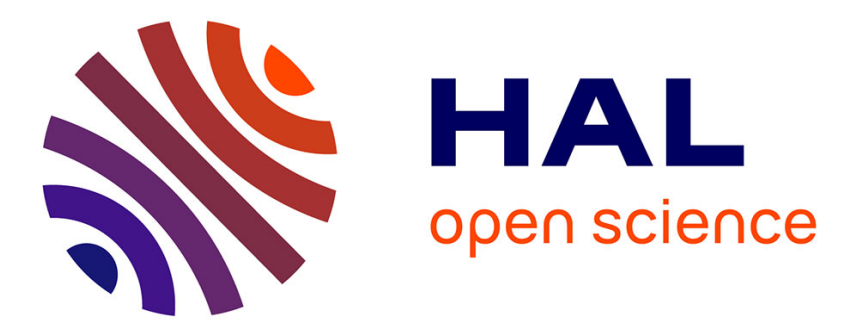

\title{
Utilisation des matières plastiques comme surface d'échange de chaleur pour économiser l'énergie dans les procédés industriels de concentration par évaporation
}

\author{
Franck Lauro, J. Huyghe
}

\section{- To cite this version:}

Franck Lauro, J. Huyghe. Utilisation des matières plastiques comme surface d'échange de chaleur pour économiser l'énergie dans les procédés industriels de concentration par évaporation. Revue de Physique Appliquée, 1982, 17 (9), pp.617-623. 10.1051/rphysap:01982001709061700 . jpa-00245039

HAL Id: jpa-00245039

https://hal.science/jpa-00245039

Submitted on 1 Jan 1982

HAL is a multi-disciplinary open access archive for the deposit and dissemination of scientific research documents, whether they are published or not. The documents may come from teaching and research institutions in France or abroad, or from public or private research centers.
L'archive ouverte pluridisciplinaire HAL, est destinée au dépôt et à la diffusion de documents scientifiques de niveau recherche, publiés ou non, émanant des établissements d'enseignement et de recherche français ou étrangers, des laboratoires publics ou privés. 


\title{
Utilisation des matières plastiques comme surface d'échange de chaleur pour économiser l'énergie dans les procédés industriels de concentration par évaporation
}

\author{
F. Lauro et J. Huyghe \\ Commissariat à l'Energie Atomique, Centre d'Etudes Nucléaires de Grenoble, Service des Transferts Thermiques, \\ 85X, 38041 Grenoble cedex, France
}

(Reçu le 21 septembre 1981, révisé le 4 décembre 1981, uccepté le 26 février 1982)

\begin{abstract}
Résumé. - On présente un procédé industriel de concentration par évaporation à multiple effet utilisant des évaporateurs à film tombant dont la surface d'échange de chaleur est constituée de gaines minces en matière plastique. Ces évaporateurs procèdent d'une conception entièrement nouvelle qui apporte une solution originale au problème de la mauvaise conductibilité thermique des matières plastiques. Un pilote de ce type d'évaporateur a fonctionné pendant quatre ans; on en rapporte ici les résultats d'exploitation.

Ce procédé, par l'économie d'investissement qu'il représente, permet de réaliser des installations plus performantes, donc plus économes en énergie.
\end{abstract}

\begin{abstract}
An industrial multiple-stage concentration by evaporation process is described; it uses falling film evaporators, the heat transfer surface of which is constituted by flexible plastic tubing. These evaporators have a new conception which brings an original solution to the bad thermal conductivity of plastic materials. A two-stage pilot unit was working for 4 years. This paper presents the results of the research program. The lessened costs of this type of evaporators permit to design less expensive industrial units; this evidently leads to improved performances, therefore to reduction in energy consumption.
\end{abstract}

1. Introduction. - La réalisation d'économies d'énergie dans l'industrie passe souvent par la réduction du coût des appareils thermiques : l'introduction de matériels moins onéreux dans les procédés permet en effet d'améliorer leur efficacité sans supplément de coût par rapport aux cycles actuels (en multipliant le nombre d'appareils de récupération de chaleur par exemple, ou en augmentant les surfaces de transfert de chaleur ou de masse), donc d'économiser l'énergie.

Le présent article décrit un procédé de concentration par évaporation à multiple-effet $\left(^{1}\right)$ (dont la mise en ouvre est fréquente dans le génie chimique ou dans l'industrie agro-alimentaire) utilisant des évaporateurs à film tombant dont la surface d'échange de chaleur est constituée de gaines minces en matière plastique. Ce type d'évaporateur, breveté par le C.E.A., permet de réaliser une économie de 20 à $30 \%$ sur le coût des investissements, car la surface d'échange est d'un prix modique par rapport à celui des surfaces d'échange thermique métalliques habituelles, donc d'augmenter le coefficient de perfor-

( $\left.{ }^{1}\right)$ Voir schéma de fonctionnement à co-courant sur la figure 1 . mance des procédés, c'est-à-dire le rapport de la quantité de produit fabriquée à la consommation d'énergie.

2. Concept d'évaporateur à surface d'échange en matière plastique. - Lorsqu'on désire abaisser le coût des surfaces d'échange de chaleur, on pense tout de suite aux matières plastiques, qui sont nettement moins chères que la plupart des matériaux métalliques couramment employés dans le génie chimique ou l'industrie agro-alimentaire, aciers inoxydables la plupart du temps, voire titane. Mais le problème de l'utilisation des matières plastiques en tant que matériau d'échange de chaleur est celui de leur mauvaise conductibilité thermique : Si on les utilise sous forme de tubes de même épaisseur que les tubes métalliques, on est obligé d'accroître la surface d'échange d'un facteur qui peut atteindre plusieurs unités, ce qui augmente énormément le volume, donc le coût des enveloppes et rend en fin de compte le matériel aussi cher sinon plus cher que le matériel «tout métallique " classique. Cette difficulté peut être tournée si l'on peut utiliser la matière plastique en épaisseur très faible, $100 \mu \mathrm{m}$ ou même $50 \mu \mathrm{m}$, sous forme de tubes de faible épaisseur que nous appellerons 
« gaines " dans la suite de l'article. On retrouve ainsi des résistances thermiques de paroi d'échange du même ordre de grandeur que les résistances thermiques des matériaux métalliques dont l'épaisseur est la plupart du temps de l'ordre du $\mathrm{mm}$ ou de plusieurs millimètres.

La possibilité d'emploi de gaines en matière plastique comme surface d'échange de chaleur existe dans les évaporateurs à film tombant utilisés dans les procédés à multiple-effet, car les différences de pression de part et d'autre de la surface d'échange de chaleur sont faibles $\left({ }^{2}\right)$ et peuvent être supportées par des matières plastiques de faible épaisseur.

Ce pas en avant n'a été rendu possible qu'en imaginant une conception nouvelle d'évaporateur à film tombant, qui est décrite ci-dessous.

Dans les évaporateurs classiques à tubes métalliques, le fluide de chauffage, généralement de la vapeur d'eau, circule dans la calandre et se condense sur la paroi extérieure des tubes en cédant sa chaleur latente au fluide à concentrer qui, lui, tombe en film sur la paroi interne des tubes. La différence de pression entre la calandre et l'intérieur des tubes est donc positive.

Une nouvelle conception d'évaporateur permettant d'utiliser les gaines en matière plastique impose d'inverser cette différence de pression, de façon que

$\left({ }^{2}\right)$ Dans les procédés d'évaporation à multiple-effet de solutions aqueuses, les écarts de température entre effets sont faibles, de l'ordre de 5 à $10^{\circ} \mathrm{C}$ généralement, ce qui entraîne des écarts de pression faibles, eux aussi. la pression la plus forte se trouve à l'intérieur des gaines (qui sont souples du fait de leur faible épaisseur) et les gonfle pour les mettre en forme : la vapeur de chauffage circule donc à l'intérieur des gaines tandis que le film de liquide à évaporer tombe à l'extérieur de celles-ci.

La figure 2 donne les schémas comparatifs des deux types de solution, métal et matière plastique.

Cette conception nouvelle d'évaporateurs à gaines en matière plastique est basée sur des dispositifs de fixation des gaines dans les plaques tubulaires et de distribution du liquide à évaporer, qui sont brevetés [1], [2].

La figure 3 présente le schéma d'une ligne d'évaporateurs de ce type fonctionnant en multiple-effet dans une version à contre-courant [3], qui est une amélioration du cycle par rapport à la version plus classique à co-courant présentée sur la figure 1 .

3. Réalisation et essais d'évaporateurs à gaines en matière plastique. - Le principe d'évaporateur précédemment décrit repose sur des résultats expérimentaux qui ont été obtenus en plusieurs étapes :

\subsection{VÉRIFICATION DE LA FAISABILITÉ DU PROCÉDÉ} sur une section d'essai monotubulaire (dont le schéma correspond exactement à la partie b de la figure 2), avec mesure des coefficients de transfert thermique sur différents types de matière plastique.

Les essais d'évaporation ont été effectués sur de l'eau à différentes températures. Les résultats obtenus avec la vapeur de chauffage de $70^{\circ} \mathrm{C}$ figurent dans le tableau I. Ils montrent que les coefficients de

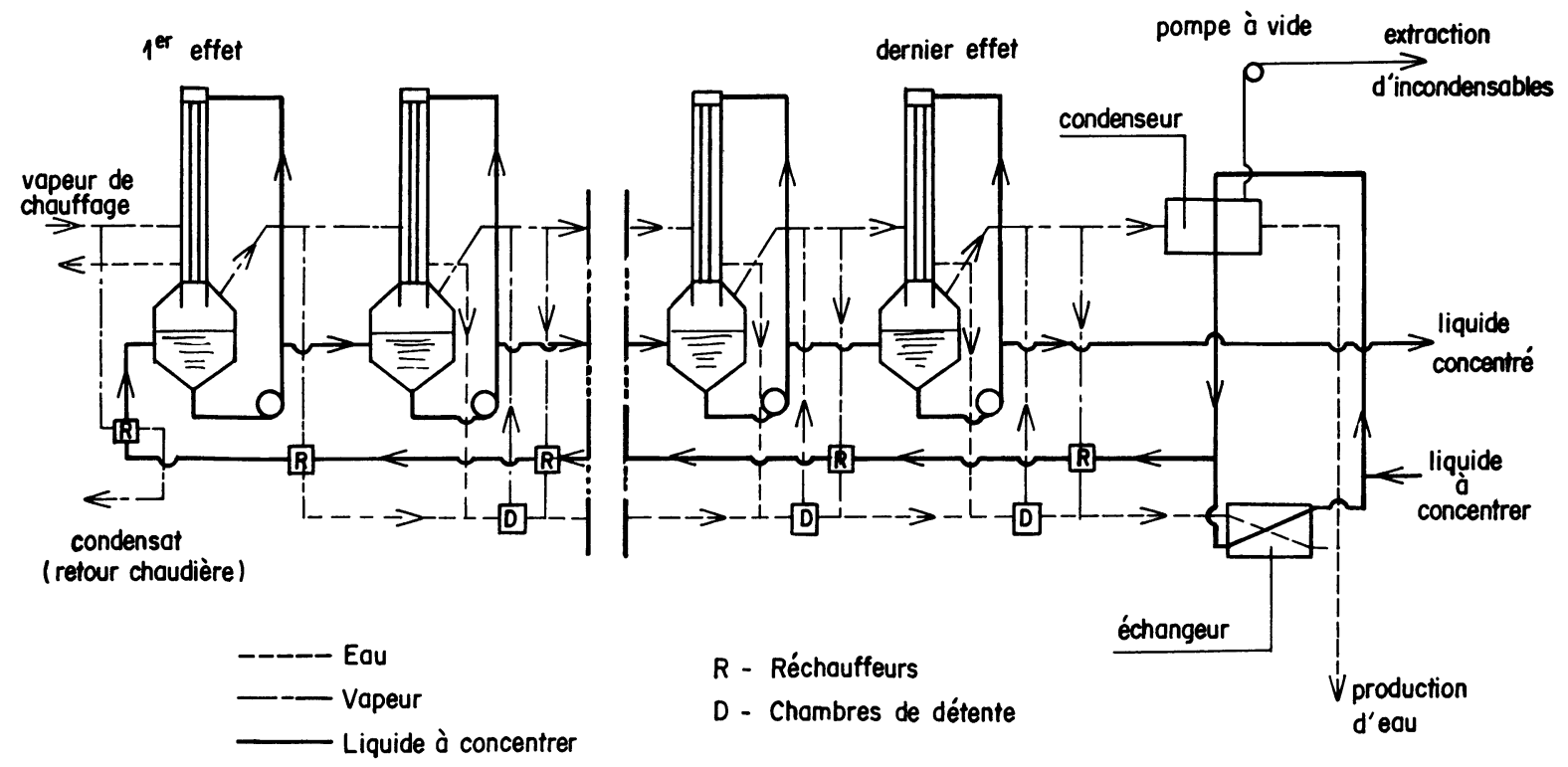

Fig. 1. - Schéma de fonctionnement (à co-courant) d'un cycle classique d'évaporation à multiple-effet utilisant des évaporateurs à film tombant à surface d'échange métallique.

[Flow sheet of a multi-stage evaporation co-current process using falling film evaporators with a metallic heat exchange surface.] 


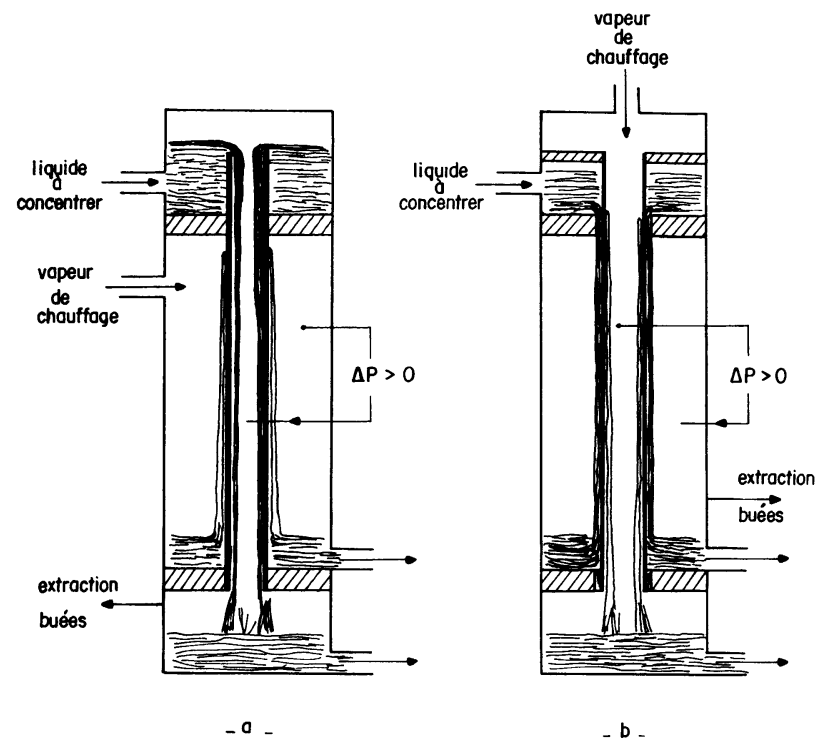

Fig. 2. - Comparaison des principes de fonctionnement d'un évaporateur à tubes métalliques $(a)$ et d'un évaporateur à gaines en matière plastique $(b)$.

[Working schemes of : $(a)$ metallic tubing heat exchange surface evaporator, $(b)$ plastic heat exchange surface evaporator.]

transfert thermique globaux (entre vapeur de chauffage et vapeur produite) sont du même ordre de grandeur, dans les mêmes conditions de fonctionnement (température, $\Delta T$, fluide), avec les gaines en matière plastique de $100 \mu \mathrm{m}$ d'épaisseur qu'avec des tubes métalliques classiques, et qu'ils sont même meilleurs lorsqu'on atteint une épaisseur de $50 \mu \mathrm{m}$ ou que l'on utilise des matières plastiques chargées en matériau conducteur (graphite par exemple).
Une explićation supplémentaire de la valeur relativement élevée du coefficient de transfert thermique au travers des matières plastiques réside dans le fait que la vapeur se condense sur les gaines principalement sous forme de gouttes, lesquelles roulent sur la paroi sans constituer le film de condensat néfaste au transfert de chaleur qui se produit habituellement sur les surfaces métalliques.

3.2 Réalisation D'Un PILOTE DE 2 efFets. - Ce pilote, dont le schéma est présenté sur la figure 4 , a été conçu et réalisé selon les principes exacts d'un appareillage à grande échelle : 12 gaines de $30 \mathrm{~mm}$ de diamètre pour un pas de $45 \mathrm{~mm}$ et $3,5 \mathrm{~m}$ de longueur, par effet; dispositifs de fixation, d'étanchéité et de dilatation conformes aux brevets. Il fonctionne avec de la vapeur d'eau comme fluide de chauffage et de l'eau de mer comme liquide à concentrer. Celle-ci a été choisie pour ses propriétés encrassantes, entartrantes et corrosives permettant de mieux cerner les possibilités du procédé et aussi à cause d'une possible application du procédé à la distillation de l'eau de mer.

Il peut fonctionner de $110^{\circ} \mathrm{C}$ à $40^{\circ} \mathrm{C}$ pour la vapeur de chauffage, avec des différences de température entre effets variables de 1 à $15^{\circ} \mathrm{C}$.

Ce pilote, dont une photographie est présentée ci-après, a fonctionné de la façon la plus satisfaisante pendant plus de 4 ans dans la station d'essais en eau de mer du CEA à Toulon. Différents types d'essais ont été conduits avec des gaines de différentes matières plastiques : mesure des coefficients de transfert de chaleur; recherche de l'influence de la présence de gaz incondensables sur les performances de transfert de chaleur; essais de prévention de l'entartrage;

Tableau I. - Coefficients de transfert thermique en écoulement à film tombant, pour différents matériaux de paroi d'échange et une température de vapeur de chauffage de $70^{\circ} \mathrm{C}$.

[Falling film overall heat transfer coefficients for different materials and heating steam at $70{ }^{\circ} \mathrm{C}$.]

\begin{tabular}{|c|c|c|c|c|}
\hline & & & \multicolumn{2}{|c|}{$\begin{array}{c}\text { Coefficient de transfert } \\
\text { thermique global } \\
\text { W/m }{ }^{2}{ }^{\circ} \mathrm{C}\end{array}$} \\
$\begin{array}{c}\text { Nature de } \\
\text { la paroi }\end{array}$ & $\begin{array}{c}\text { Conductibilité } \\
\text { thermique } \\
\mathrm{W} / \mathrm{m}^{2}{ }^{\circ} \mathrm{C}\end{array}$ & $\begin{array}{c}\text { Epaisseur } \\
\mathrm{mm}\end{array}$ & \multicolumn{2}{|c|}{$\begin{array}{c}\text { plastique chargé } \\
\text { de } 10 \% \\
\text { de graphite }\end{array}$} \\
\cline { 4 - 5 } & 25 & 1 & 3060 & \\
\hline Cu-Ni 70/30 & 0,42 & 0,1 & 3070 & 4840 \\
\hline $\begin{array}{c}\text { Polyéthylène } \\
\text { HD } \\
\text { (PEHD) }\end{array}$ & 0,13 & 0,05 & 4830 & 6800 \\
\hline $\begin{array}{c}\text { Polyvinylidène } \\
\text { Fluoride } \\
\text { (PVDF) }\end{array}$ & 0,1 & 1130 & 2060 \\
\hline
\end{tabular}




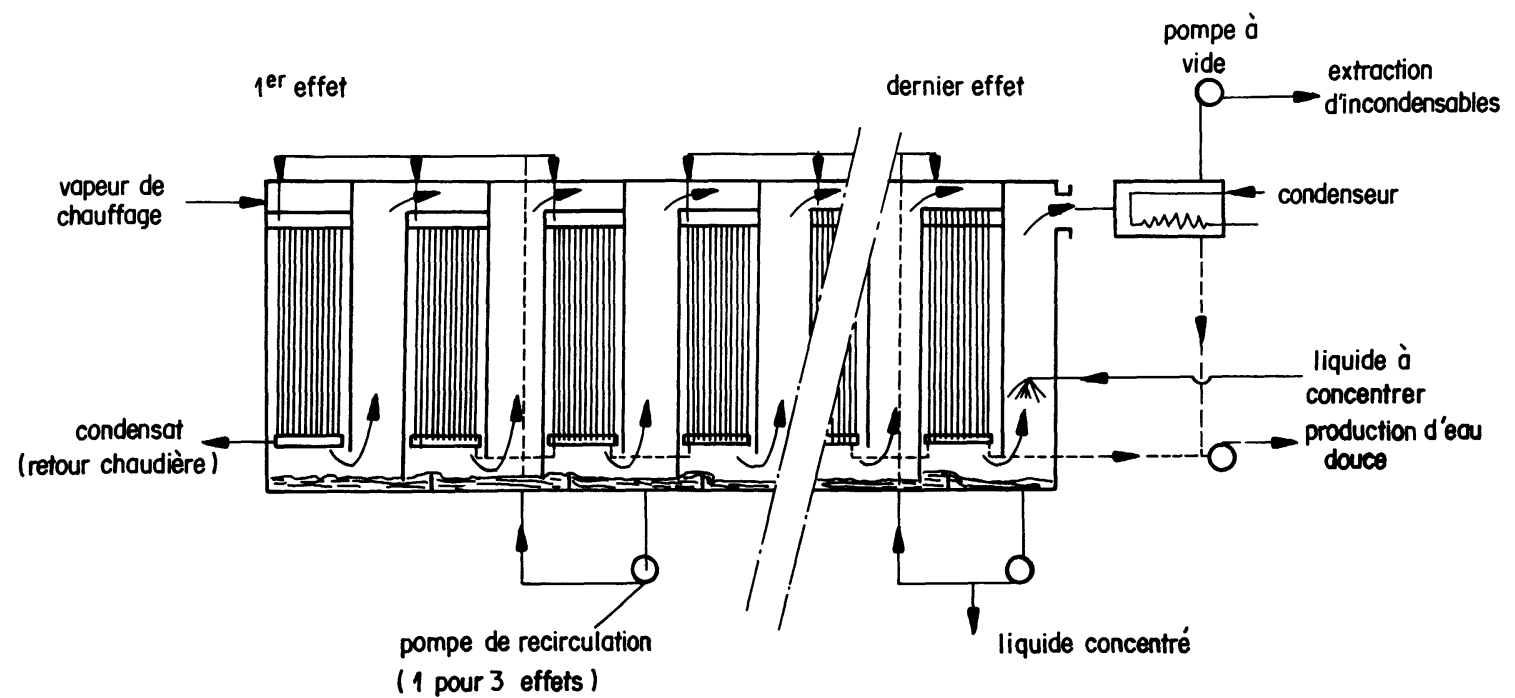

Fig. 3. - Schéma de fonctionnement (à contre-courant) d'un cycle d'évaporation à multiple-effet utilisant des évaporateurs à surface d'échange en matière plastique.

[Flow sheet of a multi-stage evaporation counter-current process using falling film evaporators with plastic heat exchange surface.]

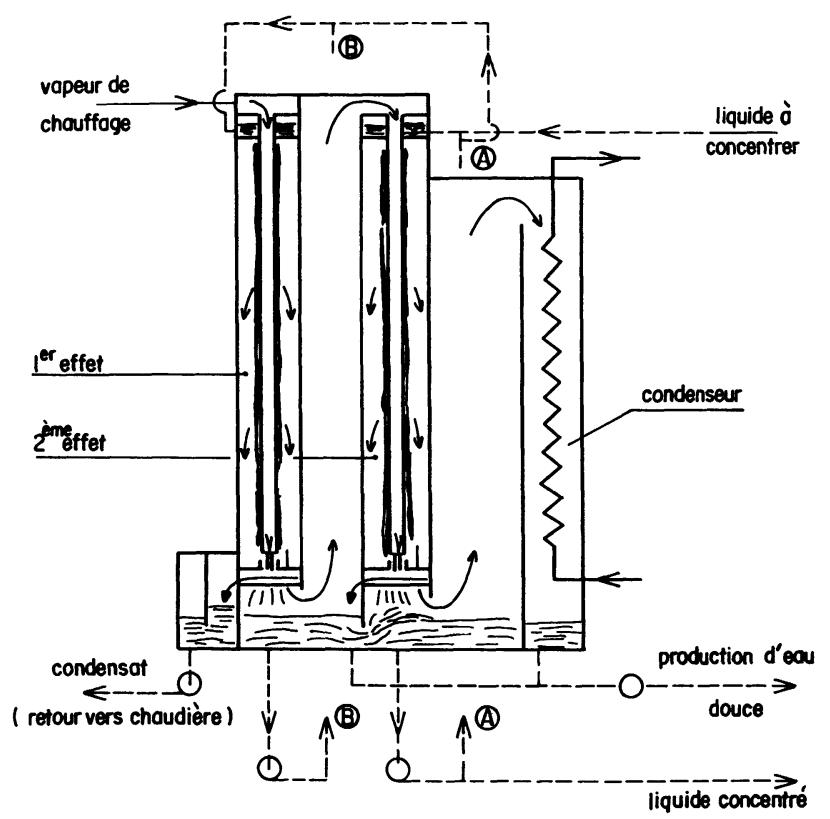

Fig. 4. - Schéma de fonctionnement d'un pilote d'évaporateur à deux effets à gaines en matière plastique.

[Flow sheet of a two-stage evaporator pilot using a flexible plastic tubing.]

essais de fonctionnement en continu et en transitoire.

3.2.1 Essais thermiques. - Plusieurs campagnes d'essais ont été effectuées avec de l'eau douce, puis de l'eau de mer non entartrante (traitée à l'acide) afin de déterminer l'influence des différents paramètres sur le coefficient d'échange thermique global :

- Température de la vapeur de chauffage : de $110^{\circ} \mathrm{C}$ à $40^{\circ} \mathrm{C}$.
- Ecart de température entre effets : de 1 à $15^{\circ} \mathrm{C}$.

- Débit d'alimentation des gaines : de 50 à $400 \mathrm{l} / \mathrm{h}$.

- Nature et épaisseur des gaines : PVDF, PEHD, POLYAMIDE 11.

- Les coefficients d'échange thermique obtenus sur le pilote confirment ceux obtenus sur la section d'essais monotubulaire, dont il était question au $\S 3.1$ en particulier les chiffres qui figurent dans le tableau I avec matériau pur, obtenus avec les valeurs suivantes des paramètres de fonctionnement : vapeur de chauffage $70^{\circ} \mathrm{C}$, écart de température $5^{\circ} \mathrm{C}$ et débit d'arrosage $200 \mathrm{l} / \mathrm{h} /$ gaine.

- On a constaté que l'influence des paramètres température, écart de température et débit d'arrosage était faible, au maximum de $15 \%$ pour les intervalles de variations considérés.

- La figure 5 donne un exemple de résultats obtenus sur l'effet du débit d'alimentation des gaines, pour une température de $70^{\circ} \mathrm{C}$ et un écart de température de $5^{\circ} \mathrm{C}$.

3.2.2 Influence de la présence de gaz incondensables sur le transfert de chaleur. - Dans les procédés industriels de concentration par évaporation qui fonctionnent presque toujours sous vide, il existe des gaz incondensables qui diminuent l'échange de chaleur de la vapeur condensante, ce phénomène étant encore plus mal connu dans les conditions de condensation en gouttes qui existent sur la matière plastique.

Cette influence des gaz incondensables a été vérifiée par un certain nombre d'essais dans lesquels on a fait varier la quantité de gaz présents dans la vapeur de façon à décider s'il était nécessaire ou non de réaliser des extractions intermédiaires d'incon- 


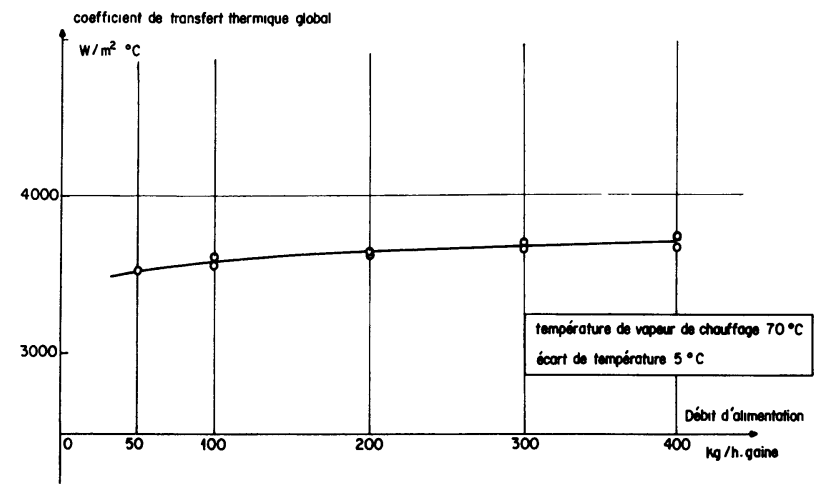

Fig. 5. - Evolution du coefficient de transfert thermique global en fonction du taux d'alimentation des gaines.

[Overall heat transfer coefficient versus falling film mass flow rate.]

densables dans une ligne d'évaporateurs à multipleeffet $\left({ }^{3}\right)$.

La figure 6 donne un exemple des résultats obtenus en faisant varier la concentration de gaz incondensables dans la vapeur jusqu'à une valeur de $3000 \mathrm{ppm}$ en masse : on constate que le coefficient de transfert thermique global est peu sensible à la présence d'incondensables dans la vapeur, et ce, jusqu'à des teneurs relativement élevées.

L'extraction classique d'incondensables, en cascade d'évaporateur en évaporateur, est donc possible.

3.2.3 Essais de prévention d'entartrage. - Des essais ont été effectués sans aucun prétraitement de l'eau de mer, liquide particulièrement entartrant dans les conditions de température où fonctionne le pilote (carbonates et sulfates de calcium, hydroxyde de magnésium).

Dans ce cas, on a constaté qu'il y avait formation d'une fine pellicule de tartre sur la gaine, mais que cette pellicule ne s'incrustait pas : périodiquement et avant que la pellicule n'atteigne l'épaisseur de $0,1 \mathrm{~mm}$, elle se détachait par plaques en conservant le tube propre, donc le coefficient de transfert thermique intact.

Malgré cela, la plupart des essais ont été conduits avec addition de produit inhibiteur d'entartrage dans l'eau de mer de façon à régulariser le fonctionnement du pilote, dont les circuits n'étaient pas adaptés à la circulation de particules solides, en quantité importante, dans l'eau. Cependant, cette propriété des gaines de ne pas s'entartrer est intéressante en cas d'incident (panne du dispositif d'injection de produit inhibiteur par exemple ou mauvais dosage)

$\left({ }^{3}\right)$ Dans les procédés utilisant des évaporateurs classiques à surface d'échange métallique, il suffit souvent d'une seule extraction d'incondensables pratiquée sur l'évaporateur le plus froid (voir Fig. 1) : les incondensables sont drainés d'évaporateur en évaporateur de sorte que leur concentration dans la vapeur croît de l'évaporateur le plus chaud à l'évaporateur le plus froid.
Coefficient de transfert thermique global

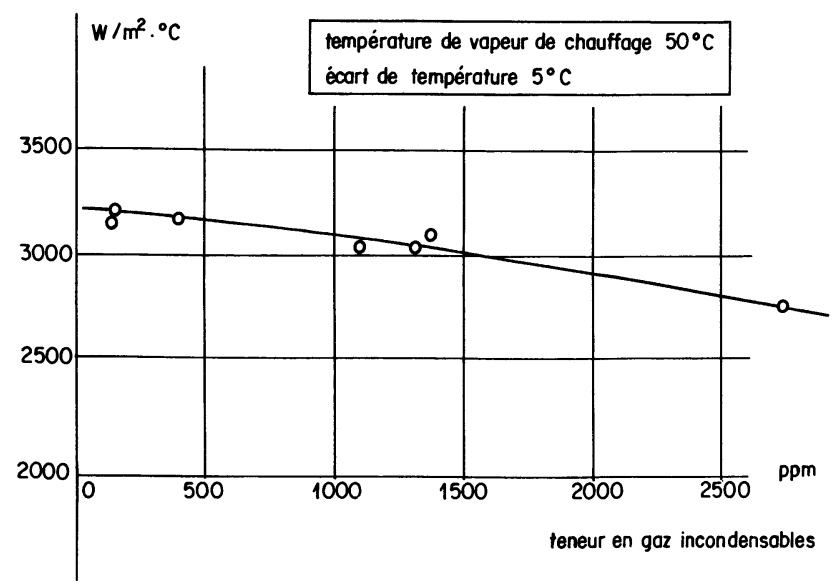

Fig. 6. - Influence des gaz incondensables sur le coefficient de transfert thermique des évaporateurs à gaines en matière plastique.

[Overall heat transfer coefficient versus non condensable gases rate.]

et pour certaines applications spéciales, à condition d'adapter les circuits (décanteurs, purges, etc...). La figure 7 donne un exemple de recherche des conditions d'encrassement en fonction du temps, pour l'application au dessalement de l'eau de mer par évaporation.

3.2.4 Essais de fonctionnement en transitoire. Différents essais simulant des incidents de fonctionnement ont été effectués : arrêt d'une ou plusieurs pompes de recirculation de liquide, augmentation ou réduction brutale de la pression de la vapeur de chauffage, etc...

Aucun de ces cas (qui peuvent être des incidents dangereux pour les unités à surface d'échange métallique) n'a eu de conséquences fâcheuses pour le pilote. Pour les différents incidents envisagés, des systèmes de régulation ou de sécurité classiques tels que vannes de régulation, soupapes de décharge, siphons d'équilibrage, ont permis à la calandre et à la surface d'échange de ne pas subir le moindre dommage.

3.3 ESSAIS MÉCANIQUES ET DE DURÉE DE VIE DES MATIÈRES PLASTIQUeS UTILISÉES. - Ces essais ont été conduits en même temps que ceux du pilote, sur des échantillons des différentes matières plastiques envisageables, placés en autoclave, sous contrainte et dans des liquides portés aux températures présumées d'utilisation. Il s'agissait d'essais de durée de vie jusqu'à rupture et d'essais d'allongement permettant de déterminer les déformations dans le temps.

Les résultats d'essais de durée de vie sont portés sur le graphique de la figure 8 , pour le polyéthylène de haute densité, donné à titre d'exemple. On voit que, la zone de fonctionnement normal des évaporateurs à multiple-effect correspondant normalement 

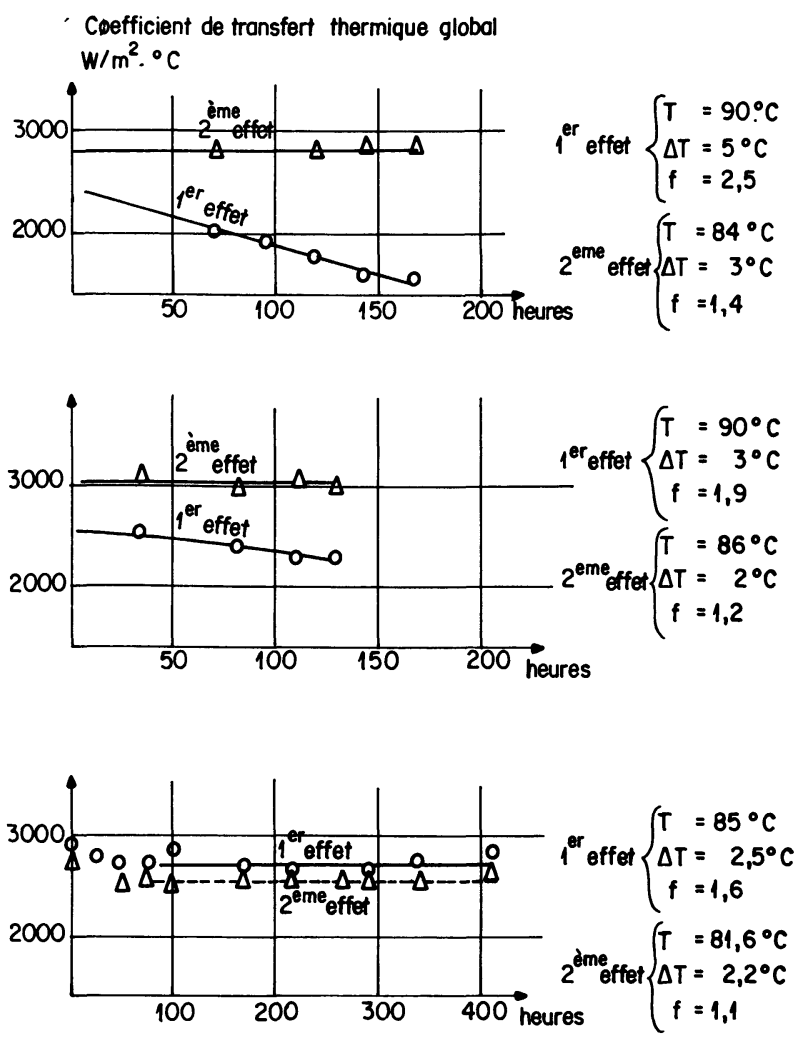

$$
\begin{aligned}
& T=\text { Température vapeur de chauffage } \\
& \Delta T=\text { Ecart de température entre effets }
\end{aligned}
$$$$
f=\text { Focteur de concentration de l'eou de mer }
$$

Fig. 7. - Evolution du coefficient de transfert thermique des évaporateurs à gaines en matière plastique en fonction du temps, pour de l'eau de mer prétraitée en polyphosphate.

[Overall heat transfer coefficient versus time, for preteated sea water.]

à une contrainte inférieure à 30 ou $40 \times 10^{-5}$ Pascal (application de la relation $\sigma=\frac{\mathrm{d} \Delta P}{2 e}$ ), la durée de vie du polyéthylène $\mathrm{HD}$ réticulé, à $80^{\circ} \mathrm{C}$ par exemple, peut atteindre plusieurs dizaines de milliers d'heures soit plusieurs années.

Les mesures d'allongement montrent en outre que les matériaux choisis ne subissent pas de déformation permanente supérieure au pourcent, dans les conditions de fonctionnement en question.

En ce qui concerne la fiabilité du système, on peut dire qu'elle est d'ores et déjà démontrée par 4 ans de fonctionnement discontinu du pilote de Toulon, par campagnes d'essais de 3 semaines, représentant plus de $10000 \mathrm{~h}$ d'essai à des températures comprises entre 60 et $90^{\circ} \mathrm{C}$, avec des gaines en PVDF.

Les progrès constants qui s'effectuent de nos jours dans le domaine de la recherche et de la mise au point de matières plastiques plus performantes permettront d'utiliser les gaines dans des conditions de fonctionnement de plus en plus sévères. A titre d'exemple,

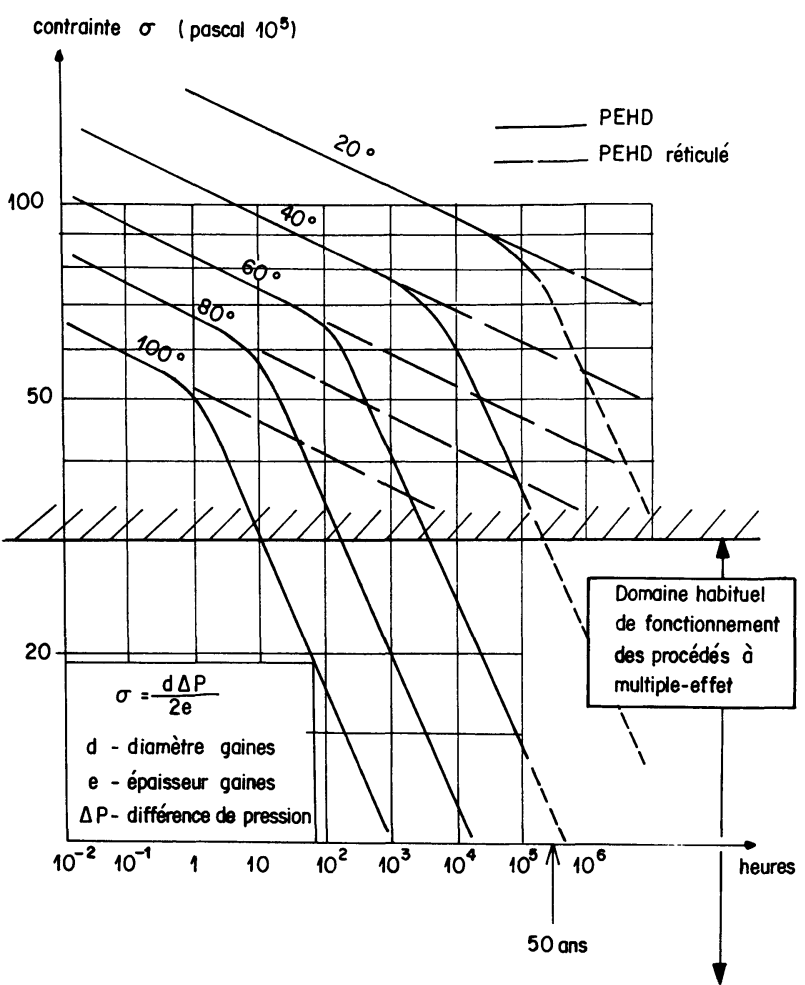

Fig. 8. - Résultats d'essais de durée de vie pour le polyéthylène haute densité (PEHD).

[Results of longivity tests for HDPE.]

on sait d'ores et déjà que le polyfluorure de vinylidène PVDF permet d'atteindre des températures de l'ordre de 110 à $120^{\circ} \mathrm{C}$.

4. Conclusion. - L'expérience acquise dans la réalisation et le fonctionnement en eau de mer, pendant près de 5 ans, d'un pilote de 2 effets conçu selon le principe d'un appareillage à grande échelle, a apporté la conviction que le principe d'utilisation d'évaporateurs à gaines en matière plastique était maintenant applicable sur un plan industriel.

Le coût minime de la surface d'échange (qui représente souvent elle-même une part importante, de 30 à $50 \%$, du coût de l'appareillage) permet d'obtenir une réduction importante, de l'ordre de $20 \%$, du coût des installations d'évaporation ou de concentration. Ce résultat permet d'évidence d'augmenter les performances des appareils à multiple-effet, donc de diminuer les dépenses en énergie pour une production donnée.

Un autre avantage important de l'emploi des matières plastiques comme surface d'échange, et sur lequel on n'a pas insisté précédemment, est l'absence totale de problème de corrosion due à l'inertie chimique des matières plastiques, ce qui peut être intéressant dans de nombreux cas, notamment en génie chimique (traitement de certains acides, par exemple) ou dans l'industrie agro-alimentaire. D'ores et déjà 


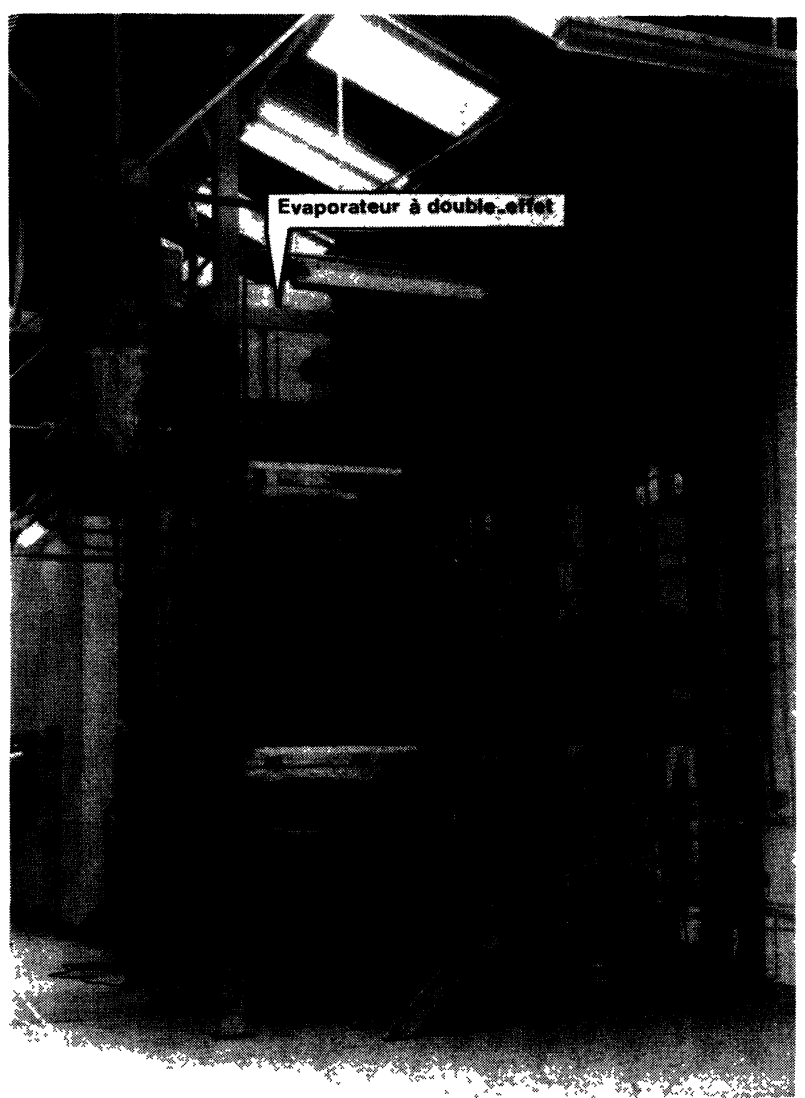

Photo. - Pilote expérimental à deux effets.

[Two-effect experimental pilot.] des applications sont envisagées pour la distillation de l'eau de mer, milieu particulièrement corrosif et entartrant, où l'utilisation d'évaporateurs à surface d'échange en matière plastique présente de nombreux avantages [3].

Les travaux se poursuivent actuellement par une étude d'avant-projet d'une installation d'évaporation de 9 effets de grosse capacité (équivalent de $1000 \mathrm{t} / \mathrm{h}$ de production d'eau) utilisant des évaporateurs de plusieurs milliers de gaines chacun.

\section{Bibliographie}

[1] Brevet $\mathrm{n}^{\mathrm{o}} 73$ 32372. Dispositif de montage de tubes en matière plastique pour évaporateurs.

[2] Brevet $\mathrm{n}^{\circ} 76$ 16047. Echangeur de chaleur à tubes souples verticaux du type à descendage.

[3] Lauro, F., 5th International Symposium on "Fresh water from the sea $\gg 2$ (1976) 261-268. Usine de dessalement à multiple-effet utilisant des surfaces d'échange en matière plastique. 